\title{
Korean Medical Students' Cognitive, Emotional, and Social Characteristics
}

\author{
Sook Hee Ryue ${ }^{1}$ and Hae Bum Lee ${ }^{2}$
}

${ }^{1}$ Department of General Education, Woosong University, Daejeon, and ${ }^{2}$ Department of Medical Education, Yeonsei University College of Medicine, Seoul, Korea

\section{국내 의학과, 의학전문대학원, 의예과 학생들의 인지·정서·사회적 특성 고찰}

${ }^{1}$ 우송대학교 교양교직과, ${ }^{2}$ 연세대학교 의학교육과

\section{류숙희 ${ }^{1}$, 이혜범 ${ }^{2}$}

Purpose: Medical schools and graduate medical schools should understand the personality and psychological qualities of graduate medical students, medical students, and premedical students and link them with the curriculum.

Methods: In this study, through June 2010 we analyzed medical papers that were published in Korea. The search terms were psychological terminology, including emotion, cognition, intelligence, social ability, stress, motivation, judgment, and learning style. Results: In the cognitive and learning aspects, preliminary doctors were under the influence of prior knowledge; cumulative learning; self-efficacy; and visual, logical, non-self-led learning types and had external learning motivation. In the emotional adaptive aspects, they appeared to be the ISTJ (introversion, sensing, thinking, judging) personality type with regard to the Myers-Briggs indicators and perfectionists, suffering from severe academic stress. Their motivation on matriculation was associated with their interests and aptitudes, and through community service, they adapted to the learning and living environment of medicine. In the social and moral aspects, they did not have high moral judgment, felt devaluated about their job than before, and tended to have an open and flexible doctor-patient relationship.

Conclusion: Medical graduate students, medical students, and premedical students have greater likelihood to cultivate their character and capacity for adaptation.

Key Words: Doctor's professionalism, Medical student's psychology, Medical education courses, Doctor's character education

서론

지난 2011년 4월 1일 전국의사국가고시와 관련해서 “예비
의사 병든 양심 $\cdots$ 의사 국가시험 조직적 유출[1]”이라는 제목 의 기사들이 일간지를 장식하였다. 이것은 예비의사, 즉 전국 의 의과대학 및 의학전문대학원 졸업반 학생들이 연합하여 국가시험문제를 몰래 빼내 배포한 내용이었고, 이 일로 서울
Received: December 7, 2011 • Revised: February 15, 2012 • Accepted: March 9, 2012 Corresponding Author: Hae Bum Lee

Department of Medical Education, Yeonsei University College of Medicine, 50 Yonsei-ro, Seodaemun-gu, Seoul 120-752, Korea

Tel: +82.10.8882.4990 Fax: +82.2.2632.7898 email: speechcom1@ hanmail.net
Korean J Med Educ 2012 Jun; 24(2): 103-115

http://dx.doi.org/10.3946/kjme.2012.24.2.103

pISSN: 2005-727X eISSN: 2005-7288

(C) The Korean Society of Medical Education. All rights reserved. This is an open-access article distributed under the terms of the Creative Commons Attribution Non-Commercial License (http:// creativecommons.org/licenses/by-nc/3.0/), which permits unrestricted non-commercial use, distribution, and reproduction in any medium, provided the original work is properly cited. 
Sook Hee Ryue and Hae Bum Lee: Korean Medical Students' Cognitive, Emotional, and Social Characteristics

지방경찰청은 전국의대4학년협의회(전사협) 회장과 집행부 학생들을 입건하였다. 이 사건을 우리는 어떻게 보아야 할까? 의과대학은 생명을 다루고, 환자의 고통과 치유에 정직한 책 임을 갖는 의사들을 양성해야 하는데, 병을 다루고 고치는 전 문지식은 가르치고 있지만, 정작 그들이 가져야 할 인성적 소 양을 가르치는 일을 소홀히 하고 있지는 않은가.

사실 의학 전문직업성이 강화되고 관련 전문직 영역이 다 종화되는 이 시점에서 현 의과대학 및 의학전문대학원의 교 육과정에서 전문직업성에 필요한 기초적 인성 및 심리적 자 질이라는 특성을 고려하여 제대로 양성해왔는가는 불분명하 다[2].

이는 의학이라고 하는 것이 오랜 기간 동안 인간의 질병에 초점을 둔 생물학적 범위에 국한되어 왔고, 의과대학 교육과 정의 내용이나 의사자격 국가시험의 평가도 이러한 생물학적 접근에 바탕을 둔 의학의 세분화된 지식에 무게를 두어 왔기 때문일 것이다. 또한 이러한 환경 속에서 의학과, 의학전문대 학원, 의예과 학생들이 의학에 대한 다양한 접근방식의 가능 성이나 의학 이외의 인문사회적 영역에 대한 관심, 의사직을 수행하되 구체적으로 어떤 전공을 선택하여 어느 곳에서 일 할 것인가와 같은 진로에 대한 고민이나 탐색의 기회가 주어 지지 않았고[2], 더하여 그들의 심리적 특징에 대한 이해와 파 악보다는 지식과 술기 중심의 전문직업의 수행력에 초점을 맞추었기 때문일 것이다.

특히 의과대학생의 경우 유급제도, 블럭식 수업, 2 주에 한 번씩 진행되는 시험, 연간 50일이 채 되지 않은 방학 등 의학 교육과정이 갖는 특수성으로 인하여 타 과 학생들보다 더 많 은 스트레스를 받는 것으로 알려지고 있다. Kim et al. [3]은 면학 저하가 생길 수도 있을 정도의 시험 스트레스를 의학과 학생들이 겪고 있다고 한다. 이처럼 의학교육과 같이 학생들 이 절대적으로 높은 학업성취도를 보이며, 대학시절에 같은 과의 거의 모든 학생들이 동일한 직업을 갖게 되는 특수한 교 육 분야에서는 의학과 학생들이 동 연령대의 다른 분야의 대 학생들과는 다른 독특하면서도 공통된 인성 특성을 가지고 있을 가능성이 많다.

요컨대 이 논문은 우리나라 의학과, 의학전문대학원, 의예 과 학생들을 대상으로 한 심리적 특성에 관한 논문들을 고찰 하여 그들의 심리적 특성을 요약하고 확인하는 기초자료를
제공하고자 수행되었다.

우리나라 의학과, 의학전문대학원, 의예과 학생들이 가진 독특한 심리적 특성을 다음과 같은 내용을 중심으로 정리하 고자 하였다.

첫째, 우리나라 의과대학생과 의학전문대학원, 의예과 학생 들의 인지·학습적 특성은 어떠한가? 구체적으로 선행지식과 누적학습의 영향은 어떠하며, 학습에 큰 영향을 미치는 자기 효능감의 정도는 어떠한가? 그리고 학습양식의 유형 및 학습 동기, 그리고 지식에 대한 태도는 어떠한가?

둘째, 우리나라 의과대학생과 의학전문대학원, 의예과 학생 들의 정서·적응적 특성은 어떠한가? 구체적으로 학생들의 성 격유형은 어떠하며, 스트레스에 대한 대처 방식은 어떠한가? 또한 학교생활 및 삶의 질에 대한 태도는 어떠한가?

셋째, 우리나라 의과대학생과 의학전문대학원, 의예과 학생 들의 사회적- 도덕적 특성은 어떠한가? 구체적으로 도덕 판단 력의 수준은 어떠하며, 의업에 대한 가치관 및 직업의식은 어 떠한가? 자신의 진로 및 성에 관해서는 어떠한 의식을 가지고 있는가?

\section{대상 및 방법}

한국의 의학과, 의학전문대학원, 의예과 학생들의 심리적 성향을 정리하기 위해서, 2010년 6월까지 한국의학교육학술 지를 비롯하여 한국의학논문 데이터베이스에 있는 의학과 학 생 관련 연구, 한국교육학술정보원에서 제공하는 한국 학술 연구정보서비스의 의학과 학생 및 의학전문대학원생을 대상 으로 한 학술지 논문, 석박사 학위논문들을 검색하였다. 검색 어는 일반적인 심리학책의 제목에 포함되는 핵심어, 즉 심리, 정서, 인지, 지능, 사회성, 학습, 스트레스, 동기, 의식, 태도, 도덕성, 성취, 성격, 성향, 적응, 의식, 판단, 생각 등을 사용하 여 검색하였다. 또한 이 검색어에 탐색되지 않더라도 의학과, 의예과, 학생 등의 키워드로 검색된 논문의 제목을 살펴보아, 위의 핵심어의 구체적 예에 포함되는 경우 분석대상 논문으 로 포함하였다. 검색은 한 연구자가 검색을 하여 논문목록을 찾고 나서 함께 목록을 살펴보고, 다른 연구자가 다시 검색하 여 추가되는 논문 목록이 없는지 확인하여 검색하는 방식을 
취하였다. 이렇게 한 이유는 국내에서 의대생의 심리를 대상 으로 한 연구가 많지 않아서 가능하면 모든 연구를 집약하고 자 하였기 때문에, 연구자 개인의 검색 순서에 따라 누락되는 논문이 있을 가능성을 없애고자 하였기 때문이었다.

살펴본 논문들 중에서 의학교육과정, 의학교수방법, 의학교 육철학, 내과·외과·산과· 소아과·정신과 등의 전공분야별 교육내용 및 교수방법 등에 관련된 연구논문은 학생들의 인 성 및 심리적 특성을 다루지 않았기 때문에 제외되고, 의과대 학생 및 의학전문대학원생의 '인지- 정서-사회적 특성'과 관 련된 논문 36편을 선정하여 분석하였다. 이 글에서 분석한 우 리나라 의과대학생의 심리를 대상으로 진행된 연구들을 검색 출처, 연구주체, 연도별(2005년 의학전문대학원 도입을 기준) 로 요약하면 Table 1 과 같았다.

분석 대상이었던 논문의 상당부분이 의과대학에서 연구된 논문들이며 나머지는 교육학과와 학회, 간호대학 등에서 연 구된 논문들이었다. 특별히 한국의학교육학회에서 나온 논문 이 가장 많은 수를 차지했다. 아울러 의학교육에서 의학과, 의 학전문대학원, 의예과 학생의 심리에 관해 관심을 가진 것은 1990년대 이후이며 본격적인 연구는 2000년 이후라는 것을 알 수 있다. 이 논문을 발행연도별로 살펴보면, 1970년부터

Table 1. Articles on Psychology of Medical Graduate Students, Medical Students, and Premedical Students

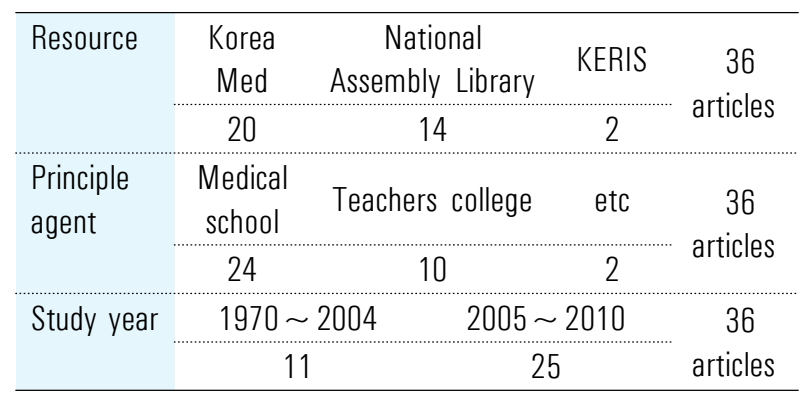

2004년까지 논문 11편, 의학전문대학원 제도가 도입된 2005 년부터 2010년 6월까지 논문은 25편이었다.

이 글에서는 심리학에서 가장 일반적으로 사용되는 내용상 의 분류인 인지·정서·사회성[4]으로 구분하는 방식을 활용 하였다. '인지'란 지적능력, 학업과 관련된 심리적 특성을 의 미하여 여기에는 인지·학업적 특성이 포함되었다. 구체적으 로 학업성취, 자기효능감, 학습유형, 학습동기, 지식에 대한 태도 등이 포함된다. '정서'는 정의적 특성으로도 불리며, 주 로 적응과 관련되는 부분들로, 이에는 성향과 성격, 스트레스 원과 대처방식, 자살 등의 위기, 학과 선택의 동기, 삶의 만족 도 등이 포함된다. '사회성' 부분은 대인관계 및 도덕성을 포 함하고 있는 개념으로, 이 논문에선 사회· 도덕적 특성으로 용어를 정리하였다. 여기에는 도덕 판단력, 가치관 및 직업의 식, 진로의식, 성의식 및 성에 대한 태도가 포함된다. 내용에 따라 분석 대상 논문의 주제를 정리해보면, Table 2 와 같이 정리될 수 있다. 즉, 인지에 관련된 논문이 13편, 정서에 관련 된 것이 14 편, 사회성에 관련된 논문이 9편이었다. 이 논문의 제목과 출처는 Appendix 1에 제시되어 있다.

\section{결과}

\section{1. 인지·학습적 특성}

의학과, 의학전문대학원, 의예과 학생들은 고등학교 혹은 대학에서 매우 높은 학업성취를 보인 학생들이다. 2010년 6 월까지 국내에서 그들의 인지, 학습적 특성을 연구한 내용을 정리하면 다음과 같다.

Table 2. Contents Classification

\begin{tabular}{lccc}
\hline & Cognitive and learning aspects & Emotional adaptive aspects & Social and moral aspects \\
\hline Contents & Academic achievement, self-efficacy, & Preference and personality, stress & Moral judgement, values and career \\
& learning style, learning motivation, & cause, crisis entrance, motivation, life & awareness, attitude toward sex \\
attitude of knowledge & 13 & satisfaction & 9 \\
Articles & & 14 & 9 \\
Total & & 36 & \\
\hline
\end{tabular}




\section{1) 선행지식과 누적학습의 영향}

의학과 및 의학전문대학원 학생들의 의과대학 내에서의 학 업성취의 특성을 정리하면 다음과 같다. 의학과 및 의학전문 대학원 학생들은 성별, 연령, 학위유형에 따라 학업성취의 차 이가 없는 것으로 나타났다. 일반적으로 의과대학에서는 여 학생이, 그리고 연령이 낮을수록, 의학전문대학원보다는 의과 대학 학생이 공부에 더 집중을 할 것이므로 높은 성취를 보일 것이라는 가정이 있지만, 이를 반박하는 증거도 있었다[5].

그러나 선행지식의 차원에서, 의학과 학생들이 언어추론 능력이 높을 때에는 의료 인문사회학과 기초임상절차에서 높 은 성취를 보였으며 자연과학추론은 기초의학 및 임상의학과 상관을 나타냈다[5]. 또한 의학전문대학원 학생의 경우 대학 학업성적은 기초의학 교육의 학업성취와 높은 상관을 보였다 [5]. 이는 의과대학 및 의학전문대학원 학생들이 가진 선행지 식의 유형이 이후의 관련 과목의 성취에 긍정적인 영향을 줄 것이라는 것을 의미한다.

또한 의학과 학생의 학업성취는 누적적인 경향이 있어서, 국내 2,371명의 의학과 학생을 대상으로 연구를 진행한 결과 의예과에서 유급을 한 학생의 경우 의학과에서도 유급을 하 는 비율이 매우 높았다[6].

\section{2) 자기효능감의 중요성}

자기 효능감은 어떤 일을 행하는 데 있어 자신이 그 일을 잘 해낼 수 있다는 믿음이다. 의예과 학생들의 자기효능감은 학업성취와 상관을 보였다. 이는 일반 학생들은 자기효능감 보다는 다른 부분, 즉 학문에 있어 긍정적 자아개념 혹은 자신 감과 높은 학업성취가 관련이 있었던 것과 상이한 결과다[7]. 그런데 의학과 학생이 의예과 학생보다 더 낮은 경향을 보였 다[8]. 그것은 의예과 공부보다 의학과 공부가 횔씬 어렵고 방 대하다보니 예과시절에 비해 '잘 해낼 수 있다라는 생각을 하 기가 힘들어진 것으로 보인다. 특히 의예과 시절 유급을 경험 했던 학생은 그 실패 경험으로 인해 의학과에서 공부를 할 때 에도 자신이 공부를 잘 해낼 수 있다는 자신감이나 믿음이 상 대적으로 낮게 나타났다[7,9]. 또한 의과대학 실습의 중요한 부분을 차지하는 객관구조화진료시험(objective structured clinical examination)에서 우수한 성적을 받는 것은 학생들 에게 술기에 대한 자신감을 심어줄 수 있어 자기효능감과 관 련이 깊은 것으로 나타났다[10].
아울러 자기효능감은 적극적인 성향이 있으면서 지속성과 노력 성향이 있을수록 높아지는 경향을 보이기에 성격적인 측면이 자기효능감과 관련 되는 것으로도 나타났다[7]. 또한 자기효능감은 의학과 학생들이 한 사람이 가지고 태어난 지 적 능력이 생애에 걸쳐 변하지 않는다는 신념을 가질수록 자 기효능감이 감소되는 경향이 있었다[11].

\section{3) 감각·시각·동화·논리적 학습 유형}

학습 유형과 관련해서는 순차적으로 공부해가는 감각형, 그림, 도표 등의 시각정보를 선호하는 시각형, 정보를 조직화 하고 모형을 만드는 동화형, 인과관계를 따져가며 공부하는 논리적 학습 유형이 있는데, 의학과 학생들은 공부를 할 때에 글 보다는 그림과 도표를 선호하고 순차적으로 혼자 생각해 가는 학습을 선호한다. 즉, 감각-시각적 성향의 학습자가 70 80\%였다[12]. 이는 다른 전공 분야나 다른 나라 의학과 학생에 비해 매우 두드러진 학습양식이다[13].

또 의과대학 의예과 학생들을 대상으로 선호하는 학습자극 과 관련된 학습양식(index of learning styles)과 지각. 기억 - 판단 시에 사용하는 정보를 조직화하는 인지양식(cognitive styles analysis)에 관해 조사한 결과, 학습양식의 분류로는 시각자(외부정보를 받아들일 때 도표, 영상 등의 시각적인 정 보를 선호하는 경우)가 $18.1 \%$ 인데 반해 인지양식에 따른 분 류로는 시각자가 $76.6 \%$ 에 이른다. 곧 의예과 학생들이 1년 넘 게 의학에 노출되면서, 인간 신체의 복잡함을 이해하는 데 시 각적인 정보가 유용함을 경험한 것이 자신을 시각형으로 지 각하는 결과를 가져온 것으로 보인다[13].

의학과 학생들의 학습양식을 보면 55\% 이상은 동화자, 즉 사물을 추상적으로 개념화하고 관찰을 통해 이해하는 학습형 태를 가지고 있고, 사람보다는 사물에 관심을 많이 가지는 논 리적인 학습 유형이다. 이에 비해 추상적으로 개념화하고 연 역적 추론을 하는 집중자와 문제해결을 위해 풍부한 상상력, 확고한 견해와 통찰력을 통해 학습하는 확산자 형의 학습자 는 적었다. 다른 전공 계열에 비해 한 쪽으로 치우친 경향이 많음을 알 수 있다[10].

일반적으로 의학과 학생들은 고등학교 시기까지 학업능력 과 학습동기가 높은 편이다. 따라서 학습내용에 맞게 자신의 학습양식을 적응적으로 바꿀 가능성이 높다. 결국 의학과 학 생들의 학습양식 정보에 기초하여 도움을 주려할 때에 그들 
이 시각자료를 활용할 수 있는 방법을 알려주는 것이 효과적 일 수 있다[14].

또한 의학과 학생을 대상으로 자기주도 학습태도를 살펴본 결과, '독창성과 새로움의 추구' 요인, '학습에 대한 자기성찰' 요인, ‘학습에 대한 애정' 요인, '학습 및 과제에 대한 효능감' 요인, '자신에 대한 긍정적 기대' 요인, '학습에 있어서의 자율 성' 요인의 6개의 하위 영역 중 ‘독창성과 새로움의 추구’ 요인 과 ‘학습에 대한 자기성찰 요인의 점수가 타 요인보다 낮은 것으로 나타났다. 그러나 그 외의 4 개 요인에서는 특정 대학 학생들이 유의하게 낮았다[15].

\section{4) 학업성취에 대한 외재적, 보상적 학습동기}

일반대학생, 의과대학생 모두 학년이 올라갈수록 학습 자 체의 즐거움을 얻기보다는 자신의 능력을 타인에게 인정받기 위한 외재적 동기에 의해 강화되는 경향이 있다[8]. 의학과 학 생들은 특히 학년이 올라갈수록 학습 자체의 즐거움(내재적 동기)보다는 보상(성적)이나 교수님께 인정받고 싶은 마음과 같은 외재적 동기로 인해 열심히 공부하는 경우가 많았다 [10].

그러나 의학과 학생들의 학업성적은 내재적 학업동기와 가 장 상관이 높은 것으로 보인다[16]. 특히 수업의 흥미는 새로 운 것을 배우려는 내재적 동기와 관련이 많았다. 그런데 숙달 하고자 하는 내재적 동기는 수업흥미와 관련이 컸으나 학업 성취도는 능력을 인정받으려는 외재적 욕구와 높은 상관을 보였다. 또한 수행목표는 수업흥미와는 관련이 적었다[10].

한편 의예과의 학생들의 경우에는 내재적 동기, 외재적 동 기 보다 학습에 전혀 동기를 보이지 않는 '무동기’가 많았다 [16]. 또한 이들의 남녀 차이를 보면, 여학생이 남학생에 비해 내적 동기, 학업성적이 더 높은 것으로 나타났다[16].

그런데 의학과 학생들이 자율적으로 동기화된 경우에는 학 업결과와 관련해서 자유의지, 행동에 있어서의 선택, 내적인 통제귀인 등을 보이지만 그렇지 않은 학생들은 압박감, 외적 인 통제귀인, 자기 자신이 무엇을 해야 하는지 구체적으로 부 여받기 원하는 행동을 원했다[7]. 즉, 의예과나 의학과에 재학 중이냐에 따라 학업에 대한 흥미와 내적 동기 및 학업성취 수 준이 달랐다. 학업성취 자체만으로는 외재적 동기에 의해 좌 우되는 경향이 있었다. 특히 의과대학생 같은 상위 학업성취 집단은 부모의 학업지원이 자기결정성 동기에 긍정적 영향을
주는 것으로 나타났다[17]. 외재적 동기를 내재화 시키는 과 정에 부모의 영향이 기여하는 것이라고 할 수 있다.

\section{5) 지식에 대한 개방적 태도}

의학과 학생들은 최면요법, 음악치료, 마사지, 요가, 기공치 료, 향기치료, 테이핑 요법 등의 보완대체의료에 대한 지식 습 득 및 이용, 관리 능력의 향상에 긍정적인 태도를 가지고 있었 고, 이에 대한 과학적 근거를 마련하기 위한 임상 실험 연구가 필요하다고 보았다[18]. 또한 태도뿐만 아니라 보건대체의학 에 대한 이해 정도에서도 더 높은 수준의 이해를 보였다. 즉, 의학과 학생들은 기존 의사들에 비해 한의학이나 기타 보완 대체 의료에 대해 더 개방적인 마음을 갖고 있다.

\section{2. 정서·적응적 특성}

의예과, 의학과 및 의학전문대학원 학생들은 일반적으로 높은 학업 스트레스에 의해 정서적으로 문제가 있을 가능성 이 있다고 지적되어 왔다. 현재까지 국내에서 그들의 정서· 적응적 특성을 연구한 내용을 정리하면 다음과 같다.

\section{1) ISTJ 성격유형이며 완벽주의적이고 이기적인 성향}

의학과 학생들의 Myers-Briggs type indicator (MBTI) 성격유형검사 결과를 살펴보면, 의학과 학생들의 성격유형은 ISTJ (introversion, sensing, thinking, judging)에서 많은 분포를 나타냈다[19]. 즉, 내향적이고, 감각형이며 사고형이 고 판단형으로서 외향적이지 않고 감정적이지 않고 지각에 의존하지 않았다[20]. 의학과 학생들은 기본적으로 객관적이 고 일관된 타당성을 인간중심 가치에 비해 더 중시하는 성격 이다.

또한 의학과 학생들은 완벽주의 성향이 있는 것으로 나타 났으며, 이는 성취목적, 학문적 효능감과 유의한 상관을 보였 다[21]. 그러나 자신에 대해 높은 기준을 설정해 놓고 일을 체 계적으로 처리하려고 꾸준히 노력하고 높게 설정된 기준에 부응하기 위해 극도로 걱정하며 자신에게 주어진 과제를 완 벽히 하지 못했을 때 습관적으로 크게 낙담하는 경향이 큰 것 은 학문적 효능감과는 부적관계에 있게 한다[21].

학생들은 대인관계 문제행동에서 통제 지배적이고, 자기중 심적이었으나 자기희생과 과관여에서 낮은 점수 분포를 보이 는 특징 또한 있었다[22]. 


\section{2) 학업스트레스와 문제중심·회피형 대처}

의학과 학생들은 대학 입학연령, 평균 자습시간, 취미, 종교 에 따라 스트레스 수준이 달랐으며, 학년, 흡연도 스트레스와 유의미한 상관이 있었다[23]. 특히 의학과 여학생은 주로 의 과대학 생활과 분위기 때문에, 남학생은 공부 양의 부담 때문 에 스트레스가 많다고 하였다[24]. 여학생이 학업스트레스가 더 크다고 지각한 연구도[16] 있었다. 남자 의학과 학생은 흡 연, 음주 등이 영향을 미쳤지만, 여자 의학과 학생에게는 거의 영향을 미치지 않았다[25]. 학업에 관심이 없는 의학과 학생 이 자신의 학업성적을 낮게 지각하고 일반적인 학업스트레스 는 물론 의학공부에 대한 스트레스도 더 많이 겪는 것으로 나 타났다[16].

의학과 학생들은 일반학생들보다도 우울증 비율이 높은데, 그것은 학업 스트레스, 억압적(위계적) 의대 환경 등에 기인 한다고 보여진다[26,27]. Kim et al. [3]은 특히 시험 스트레 스는 의학과 학생들에게 상당한 스트레스로 작용하고 있고, 이로 인해 부분적인 면역 저하가 올 수 있다고 발표했다. 그런 데 의학전문대학원 학생들의 심리적 안정성은 의학과 학생보 다 상대적으로 좋았다[28]. 의학전문대학원 학생들이 의학과 학생들보다 상대적으로 연령대가 높고 사회 경험이 많고 '의 학이라는 학문을 뒤늦게 선택하여 그 만큼 열정과 애착이 큰 것으로 보인다[28].

의학과 학생들이 자주 활용하는 스트레스 대처방식은 문제 중심 및 회피의 대처전략인데, 이는 문제를 해결하고자 하는 태도와 동시에 문제를 피하거나 잊어버리려고 노력하는 방식 이다. 이는 건강한 적응력을 가지고 있지만 불안수준이 높은 특성을 보여준다[28].

\section{3) 자살의 위기}

인간의 발달 시기 중 대학생 시기인 청년기는 신체적인 성 숙이나 정신적인 발달 등 심신양면에 걸쳐 어린이에서 어른 으로 전환이 완성되어가는 시기이다[29].

의학과 학생들의 Minnesota multiphasic personality inventory (MMPI)에서도 1980년도에서 1992년도의 학생들을 보면 정상 범위에 있기는 하지만 정신장애를 보이는 경향과 건강염려증 등이 높은 편이었다[30]. 또한 학업스트레스에 의 해 우울증에 빠진 의학과 학생들이 많으며, 이는 학업과정에 서 적절히 대처하기 힘들며 그 결과는 작게는 학업 실패부터
극단적인 경우 자살까지 나타날 수 있다는 우려를 낳고 있다 [26,27].

\section{4) 적응에 영향을 주는 의과대학 선택 동기와 전과 희망}

의학과 학생들이 의과대학 지원을 결정한 시기는 고등학교 후반기 혹은 재수할 때가 가장 많았다. 지원 동기는 인류에 대 한 봉사가 가장 많았고(37 42\%), 지원결정 시 외부의 영향을 받은 경우가 많았다(38 47\%). 가족 중 보건의료인이 있는 경 우 그들에 의해 의과대학 선택 동기를 부여받았다(52 58\%). 의과대학에의 진학만족도는 지원 결정시기가 이를수록, 지원 동기가 인간에 대한 봉사와 적성에 적합한 경우 그리고 지원 결정 시 본인의 의사가 많이 반영될수록 높았다[31]. 의학전 문대학원 학생들은 의학을 선택하는 경우 봉사와 과학적 흥 미를 더 중요한 것으로 생각하는 경향이 있었다[32].

이와 같은 의대 선택동기는 학업과 관련이 있었는데, 사회 경제적 안정성을 이유로 의과대학을 선택한 학생들은 다른 이유로 선택한 학생보다 쉬운 난이도의 공부를 선호했으며, 이는 부모와 주변의 권유로 진학한 학생보다 더 낮았다[7].

또한 의과대학 지원 동기가 우발적 선택과 사회적 안정 등 목표 혼란형 학생의 반 이상이 적응상의 문제를 나타냈다. 이 는 목표지향형과 성취지향형의 학생들이 적응상의 문제를 보 이지 않았다는 것과 대별된다. 고등학교 계열, 입학전형방법 에 의한 의과대학 적응도의 차이는 나타나지 않았으며 지원 동기가 영향을 주는 것으로 나타났다[33].

의과대학생 중에서 전과를 원하는 학생이 $5.8 \%$ 였으며, 대 학진학기회가 다시 주어질 경우 의과대학 입학을 희망하지 않는 경우가 22 27\%나 되었다[31].

\section{5) 심리적 삶의 질과 사회적 삶의 질}

의학과 학생들의 수면, 휴식, 성적 활동, 레저와 여가 활동 에 대한 참여와 기회는 일반대학생에 비해 낮은 반면, 약물과 치료에 대한 의존도, 경제적 자원, 새로운 정보와 기술을 얻을 수 있는 기회는 더 많았다[27].

또한 남자 의학과 학생이 여자 의학과 학생보다 삶의 질이 높게 나타났다[34]. 나아가 1주일에 1 3시간의 운동을 하는 경우와 특수목적고등학교(특목고) 출신인 경우 삶의 질이 더 높게 나타났다[34]. 그러나 등교 거리, 출신고 소재지, 거주형 태, 입학형태, 종교, 흡연, 커피, 수면시간, 이성교제, 서클, 친 구, 용돈 등은 의학과 학생의 심리적, 사회적 삶의 질에 영향 
을 미치지 않았다[34]. 사회적 관계 영역에서는 의과대학생의 삶의 질이 일반대학생보다 유의하게 낮았다[27].

그러나 의과대학 재학에 대한 긍지, 직업의 향후 전망을 밝 게 보는 의학과 학생일수록 대학생활의 만족도가 높았다[35]. 특히 내적 동기에 의해 의대에 입학한 의학과 학생일수록 사 회적인 전망에 의해 입학한 학생보다 심리적 삶의 질이 더 높 았다[34]. 또한 실제 생활만족도, 학생생활 적응도가 높은 학 생이 자신감이 많았다[7].

\section{3. 사회·도덕적 특성}

의학과 및 의학전문대학원 학생들은 예비의사로서 사회적, 도덕적 측면에서 건전한 성향을 가져야 하고, 그것을 성장시 킬 기회를 제공받아야 한다. 현재까지 국내에서 이들 의학과, 의학전문대학원, 의예과 학생들을 대상으로 그들의 사회·도 덕적 특성을 연구한 내용을 정리하면 다음과 같다.

\section{1) 도덕판단력}

의학과 학생들의 도덕적 판단력을 보면 의학과 학생들은 인습수준인 4단계의 법과 규칙위주의 도덕판단을 하는 경향 이 가장 많았다. 이는 간호대학생들이 인습수준보다 높은 사 회계약수준인 $5 \mathrm{~A}$ 수준의 도덕 판단력을 가장 많이 한 것과 비교된다[36]. 또한 의과대학생의 도덕 판단력은 예과에 비해 떨어지는 경향을 보였다[37]. 사회정치적 추론능력은 학년의 증대에 따라 성장되었지만, 의료행위의 이점이나 높은 수입 에 대해 기대가 많고 보수적인 학생일수록 의사들의 이익에 관련된 사회경제적인 문제나 자율성 등에서 더 큰 관심을 가 졌다[38].

의사는 감염 위험을 무릅쓰고 치명적인 전염병에 걸린 사 람을 치료할 도덕적 의무가 있다는 질문에 대해 미국에서 94\%가 동의하는 데 비해 우리나라에서는 의과대학생의 $58 \%$ 가 동의하였다[39]. 의학과 학생들의 도덕민감성에서 의사역 할채택, 타인복지, 사태지각, 결과예측, 환자역할채택, 민감성 의 하위 다섯 영역들에서 학년별로 상이한 양상을 보였으며, 중간학년에서 가장 높았다[37].

\section{2) 의업에 대한 가치관 및 직업의식}

의학과 학생들은 전문직업과 관련된 가치에서 인간, 과학, 적성 중에서 인간에 대한 봉사를 가장 중시하는 경향이 있었 다[38]. 또한 의학전문대학원 학생들은 의예과 신입생에 비해
사회적 책무성을 더 중시하고 의료 외의 분야에도 높은 관심 을 갖는 것으로 나타났다[39].

전문직업과 관련된 의식을 보면 의과대학생들은 급속한 의 료 환경의 변화 속에서 의사의 사회적 지위와 자율성이 하락 하며 의사의 노력에 대해 사회가 평가 절하하는 것으로 인식 하였다. 그러나 사회의 부정적 평가의 책임이 의사들에게도 있으며 결국 개인적 차원과 집단적 차원에서 노력이 필요하 다는 점을 인식하고 있는 것으로 나타났다. 특히 의사 집단의 가장 큰 문제점으로 지적되었던 폐쇄성과 배타성에서 벗어나 보다 적극적인 사회와의 의사소통이 필요하다고 지적하며, 개인적으로는 소비자 중심적인 의료 환경에 적응하기 위해 개방적이고 유연한 의사-환자 관계를 형성하려는 경향을 보 여주고 있다[40].

의사가 수입이 많고 존경을 받는 직업이라는 생각을 가진 학생일수록 사회와의 관계에서 불만을 가지고 있었고, 전통 적인 의사-환자 관계를 지향할수록 배타적인 경향을 보였지 만, 개방적인 의사-환자 관계와 직업의식을 가진 학생들은 보 다 유연하고 긍정적인 사회의식을 보여주었다[40].

의학과 학생들은 자신의 전문성에 대한 평가에서 졸업반이 된 뒤에도 의학적 지식, 기타 전문지식에 대해 자신이 없다고 반응하였으며, 교육과정에서 다루지 않는 전문적 태도영역을 그들의 실제 태도수준과 상관없이 매우 높게 평가하는 경향 이 있었다[40].

\section{3) 진로의식}

의학과 학생들은 자신의 직업 및 분야선택 등의 진로를 결 정할 때에 신중하고 주체적인 경향을 보이며, 지원 학교의 사 회적 평판보다는 교육 및 연구 역량, 수련병원의 역량처럼 질 적인 부분을 중시하였다[39]. 장래희망에서 연구직 교수를 원 하는 학생의 수가 점차로 늘어가고 있는데, 특히 임상의학교 수 희망 비율 $44.6 \%$ 는 미국 $15.4 \%$ 에 비해서 훨씬 높은 경향 을 보이는 것이었다[39].

\section{4) 성의식 및 성에 대한 태도}

의학과 학생들의 성의식 및 성 태도를 보면 간호대학 등의 일반대학생들은 연령, 거주형태, 건강상태에 따라 차이를 보 였지만 의학과 학생은 차이를 보이지 않았다[25]. 성경험을 보면 일반대학생들은 연령, 성 태도, 거주형태 등에 따라 차이 가 있었는데 의학과 학생은 차이가 없었다[25]. 이는 의학과 
Sook Hee Ryue and Hae Bum Lee: Korean Medical Students' Cognitive, Emotional, and Social Characteristics

학생들 간 성의식 및 태도, 행동에 있어 편차가 크지 않음을 보여준 결과였다.

\section{고찰}

의과대학이나 수련 병원은 여전히 의술 그 자체에 뛰어난 의사 양성에만 초점을 두고 있을 뿐 '진정한 치료자를 길러내 기 위한 인술을 펼칠 의사를 양성하는 데에 적극성을 보이지 못하고 있다. 사실 의과대학 교육에서 초점을 두어야 할 것은 최첨단 의학의 기술이나 세부적 지식을 넘어 의학이라고 하 는 고유한 직종에 맞는 기초자질을 함양시키는 것이다[41]. 의학과, 의학전문대학원, 의예과 학생들의 심리적 특성을 정 확히 이해하고 그 특성을 고려하여 교육할 때 이들이 훗날 의 사로서 전문 직업성과 높은 도덕성을 갖고 진정한 치료를 행 할 수 있는 바람직한 의료인으로 성장될 수 있을 것이다. 의학 과, 의학전문대학원, 의예과 학생들의 심리적 특성을 분석한 이 연구의 내용을 요약하고 교육적 함의를 정리하면 다음과 같다.

첫째, 인지·학습적 측면을 정리해보면, 의학과, 의학전문대 학원, 의예과 학생들의 학업성취 특성은 선행지식의 정도에 큰 영향을 받으며, 성공의 경험과 그로 인한 자기효능감의 영 향을 많이 받는 것으로 나타났다. 또한 학습 유형은 감각. 시 각적- 동화적· 논리적 학습 유형을 가지고 있었다. 지식에 대 한 태도에 있어 개방적인 태도를 가지고 있다.

의학과, 의학전문대학원, 의예과 학생들의 인지· 학습적 측 면을 고려한 교육적 함의를 정리하면, 의예과 시절부터 의학 과 공부를 지속할 수 있는 선행지식을 갖도록 지도할 필요가 있으며, 의과대학에서의 작은 성취일지라도 성공을 반복적으 로 경험하여 자아효능감을 높일 기회를 가져야 한다. 또한 학 습자료를 위해서 시각적 단서를 포함시키고, 관찰의 기회를 주고, 논리적으로 생각할 기회를 주는 것이 도움이 된다. 또한 동기 부분이 보다 더 내재화되도록 도와줄 필요가 있다.

또한 학업성취도가 내재적 동기에 의해서 움직일 수 있도 록 평가방식을 개선하는 것이 필요할 수 있다. 동기가 내재화 되면 자기결정성이 커지고[17], 학업에 있어 자기주도성이 향 상될 것이다. 최근의 자기결정성이론(self-determination theory)에 따르면 내재적 동기와 외재적 동기는 연속선상의 개념으로 파악하여 외재적 동기는 내재적 동기로 발전될 수 있다고 예측한다[42]. 이와 같은 관점에서는 외재적 동기를 내재적 동기로 옮아갈 수 있도록 지도하는 방안을 고려할 수 있다.

둘째, 정서·적응적 측면을 정리해보면, 의학과, 의학전문대 학원, 의예과 학생들은 MBTI 성격유형 중에서 ISTJ로서 내 향적이고 감각적이며 사고와 판단경향의 성격을 가진 학생들 이 많다. 또한 관계에 있어서 자기중심성이 강하고, 다소 이기 적인 측면이 있다. 이와 같은 성향은 과중한 학업스트레스에 의해 더욱 심화될 가능성이 있으며, 의학과 학생의 스트레스 대처 전략은 주로 문제중심적이면서도 회피형 경향으로 불안 한 상태이다. 그러나 의과대학 및 의학전문대학원을 선택한 동기가 의학자체에 대한 흥미와 적성 및 봉사와 관련이 될 때 에 비교적 더 잘 적응하는 것으로 나타났다.

이와 같은 결과를 통한 교육적 시사점을 정리하면, 의학과, 의학전문대학원, 의예과 학생들은 자신의 적응은 물론 졸업 후 환자와의 관계 형성에 있어서도 자아 중심적 성향을 벗어 나기 위한, 교육경험들이 필요하다. 예를 들면 교육과정 속에 서는 여러 친구들과 함께하는 협동학습 등을 통해, 그리고 학 과 외 학생지원 프로그램으로서 평생학습을 하는 의사로서, 긴급하고 때로는 과중한 의업을 수행하며, 환자에게 더 따뜻 하고, 관심을 가지는 의사 및 교수모델들을 만나고 관찰할 기 회를 갖는 것이 도움이 될 것이다.

또한 의학과, 의학전문대학원, 의예과 학생들은 일찍부터 스트레스 관리 능력을 갖도록 훈련되어야 한다. 스트레스를 적절하게 관리하였을 때 면역력이 증가하고, 우울과 불안이 줄어들었으며, 동정심이 증가되었고, 선택적 치료에 대한 지 식이 증가하고, 스트레스의 효과에 대한 지식이 늘어났으며, 충돌을 더 잘 해결하게 되었다고 한다[27]. 의과대학에서 주 기적인 상담과 교육을 시행하는 것이 과도한 학업과 위계적 의과대학 환경, 기타 여러 스트레스로 우울증에 걸린 의학과 학생들에게 긍정적 영향을 미칠 수 있을 것이다[27]. 또한 의 과대학에의 적응은 의과대학 선택 동기에서도 영향을 받기 때문에 선택의 동기가 의학 자체의 흥미, 봉사, 적성이 있지 않은 학생들의 경우 전과, 동기의 재조정 등의 개별 지도가 필 요해 보인다. 
셋째, 사회· 도덕적 측면을 정리해보면, 의학과, 의학전문대 학원, 의예과 학생들의 도덕 판단력이 기대에 미치지 않는 것 으로 나타났다. 오히려 같은 공간에서 근무할 간호사들의 도 덕 판단력보다 낮은 경향이 있었다. 의사로서의 직업에 대해 서 학생들은 사회적 지위와 자율성이 감소한 것으로 인식하 였지만, 이전 세대보다 폐쇄성과 배타성에서 벗어나 보다 개 방적이고 유연한 의사-환자 관계를 형성하려는 경향을 가지 고 있었다. 또한 의학과 학생들은 많은 시간을 할애하는 의학 지식에는 가장 자신이 없어했으나 교육과정에서는 거의 다루 지 않는 전문적 태도영역에서는 매우 높은 자신감을 보였다. 이들 결과를 통해 얻을 수 있는 교육적 시사점은 의학과, 의 학전문대학원, 의예과 학생들의 도덕 판단력 및 사회추론능 력, 전문성 교육은 그들 자신의 인식보다는 낮은 수준에 있기 때문에 이를 더욱 강화시킬 필요가 있다는 것이다. 새로운 시 대 변화에 따른 의사가 가져야 할 바람직한 의사-환자 관계, 개방성 그리고 전문적 태도에 관해서 학생들의 사고의 폭을 넓히는 더욱 열정적인 노력이 필요할 것이다.

이 연구가 의학과, 의학전문대학원, 의예과 학생의 심리적 특성을 다룬 국내의 많지 않은 연구들을 토대로 정리된 논문 이며, 일개 의과대학 및 의과대학원에서 이루어진 연구들이 많아서 연구의 결과를 일반화하기에는 한계점이 있다. 그러 나 의학과, 의학전문대학원, 의예과 학생들의 인지, 정서, 사 회적 특성은 향후 좋은 의사로 성장하기에 바람직한 측면과 우려되는 측면을 추정하는 데에는 도움이 될 것이다. 구체적 으로 바람직한 측면으로는 지식에 대한 개방성과 시대흐름에 따라 직업인인 '의사'에 대해 새롭게 받아들이려는 경향을 지 적할 수 있다. 그러나 의학과, 의학전문대학원, 의예과 학생들 이 가진 학업, 성격유형, 정서특징, 사회성 경향은 그들이 처 한 과중한 학업과 관련하여 우려스러운 면이 있어서, 앞으로 보다 더 긍정적이고 협동적인 성향을 기르도록 도와줄 필요 가 있다. 또한 의사로서의 윤리의식과 책임의식도 좀 더 성장 시킬 필요가 있다.

의학과 학생들의 심리적 특성을 다룬 외국 연구들을 살펴보 면, 우선 미국의학협회(The American Medical Association, AMA)에서 발행되는 저널인 'JAMA' (http://jama.amaassn.org)와 미국의과대학협회(The Association of American Medical Colleges, AAMC)에서 발행되는 저널인
'Academic Medicine' (http://journals.www.com/academicmedicine/ pages/default.aspx)에서 "의대생들 특성”(medical student characteristics)에 관한 연구를 살펴보니 국내 연구들 에 비해 양적으로 월등히 많았으며 주제 역시 다양하였다. 특히 의과대학 입학과 졸업, 전공 선택에 있어 인구사회학적 특성과 의 관련성, 종합적인 직업개발 프로그램을 받은 학생들의 학교 생활 만족도 의대 졸업생들의 직업 선택의 문제, 즉 '누가 미래 의 외과 의사가 되는가?와 같은 어떤 전공에 어떤 특성의 학생 들이 많이 지원하며 실제 직업 및 전공 만족도에 있어 어떤 특성의 학생들이 만족도가 더 높은지, 또한 학생들의 특성과 직업(의대 지원과 전공 선택)과의 연관성을 다룬 연구들이 많았 다. 이것은 프로페셔널리즘을 강조하며 직업에 대한 소명의식 을 중요하게 생각하는 미국에서 의과대학 학생들 각자가 자신 의 특성에 맞는 전공을 선택하고 좀 더 만족하며 일할 수 있도록 도움을 주기 위해 노력하고 있는 것으로 보인다.

또한 유럽의학교육학회에서 발간한 국제학술지인 'Medical Teacher' (http://informahealthcare.com/loi/mte)에서 "의 대생들 특성"에 관한 연구는 약 187 편이 검색이 되었다. 그 중 에서 의대생들의 인지·정서·사회적 특성을 다룬 연구들은 총 33편이며, 구체적으로 1970 1990년에 1편, 1991 2000년 에 3편, 2001 2010년까지 23편, 2010년 이후인 2011년 한 해 동안 6편이 출간되었다. 의대생들의 심리적 특성에 관한 연구 가 2000년 이후에 본격적으로 이루어졌다는 것은 국내 연구 와 공통점으로 보인다. 반면 이번 연구에서 분석한 국내 연구 들과의 차이점은 국내 연구들에 비해 상대적으로 의과대학 졸업 후까지 바라보는 종단 연구나 질적 연구가 많았다. 또한 국내 연구들에 비해 외국 연구들은 학습동기나 학업성취 같 은 '학습에 관한 연구보다 프로페셔널리즘(직업 전문성)과 환 자 중심 태도, 문화적 다양성 등에 대한 인식과 태도에 관한 연구가 많이 이루어졌다.

앞으로 국내에서도 의학과, 의학전문대학원, 의예과 학생을 대상으로 한 좀 더 다양한 연구, 구체적으로 사회에 대한 관 심, 의료의 사회적 가치에 대한 민감성, 선후배관계 및 친구관 계와 적응, 바람직한 여가생활, 의사로서의 자아개념의 발달, 환자에 대한 태도, 직업윤리 등의 더 다양한 주제를 다룬 연구 들이 이루어진다면 더 좋은 의과대학 및 의과대학원의 프로 그램을 구성하는 데 좋은 기초자료가 될 것이다. 
Acknowledgements: None.

Funding: None.

Conflicts of interest: None.

\section{REFERENCES}

1. Paek MK. Yebi uisa byeongdeun yangsim: uisa gukgasiheom jojikjeok yuchul [Internet]. Seoul, Korea: Seoul Newspaper; 2011 [updated 2011 April 1; cited 2011 April 10]. Available from: http://www.seoul.co.kr/ news/newsView.php?id=20110401010014.

2. Kim S. Current trends in medical education by analysis of journals of medical education. Korean J Med Educ 2004; 16: 109-117.

3. Kim CS, Shin SC, Gang MH. Effect of the test stress on the immunological functions of the medical students. Chungnam Med J 1995; 22: 423-436.

4. Myers DG. Psychology. 9th ed. New York, USA: Worth Publishers; 2009.

5. Lee SJ, Choi W, Kim SY, Choi JW. Correlation of academic achievements with cognitive admission variables and demographics at Chungbuk National University Graduate Medical School. Korean J Med Educ 2009; 21: 59-66.

6. Yu SH, Nam CM, Kim SI, Oh HJ. Cohort analysis on academic achievement of medical students. Korean J Med Educ 1994; 6: 20-28.

7. Lee YM, Ham BJ, Lee KA, Ahn DS, Kim MK. The relation of self-efficacy with environmental factors, personality and academic achievement in medical students. Korean J Med Educ 2006; 18: 249-257.

8. So Y. The effects of achievement goal orientation and self-efficacy on course interests and academic achievement in medical students. Korean J Med Educ 2008; 20: 37-49.

9. Hur Y. Are there gaps in the perception of medical students' professionalism between medical students and professors? Korean J Med Educ 2008; 20: 209-217.

10. Kim SH, Roh HR. The assessment of medical students' learning styles using the Kolb learning style inventory. Korean J Med Educ 2007; 19: 197-205.

11. Ahn D, Lee Y, Baek KJ, Chung SI. Predictors of medical students' beliefs about intelligence: affective and cognitive factors, parental interest, and home economic status. Korean J Med Educ 2008; 20: 219-229.

12. Yeo SH, Lee KW, Chang BH, Kang E, Kang DS, Kwak YS, Kim BW, Lee YC, Lee JM, Cho HJ, Chung JM. Learning styles in medical school. Korean J Med Educ 2006; 18: 31-40.

13. Kim M, Kim S, Lee J, Ahn D, Lee Y. Characteristics of medical students' learning styles and their relationships to GPA. Korean J Med Educ 2006; 18: 133-140.

14. Ahn S, Kim M, Ahn D. Relationships between cognitive and learning styles of premedical students. Korean J Med Educ 2010; 22: 7-13.

15. Chun KH, Park WK, Lee SS, Park YS, Kang E. A study on the educational climate, self-directed learning and creative thinking in medical school. Korean J Think Dev 2010; 6: 179-200.

16. Ahn D, Park G, Baek KJ, Chung SI. Academic motivation, academic stress, and perceptions of academic performance in medical students. Korean J Med Educ 2007; 19: 59-71.

17. Shin JH, Jin SJ, Kim YJ. The influence of perceived parental academic support, achievement expectation and daily control on children`s self-determination motivation according to their academic achievement level. Korean J Educ Psychol 2010; 24: 121-137.

18. Kim HJ. Knowledges, attitudes and beliefs on complementary and alternative medicine among medical students and oriental medical students [dissertation]. [Seoul, Korea]: Seoul National University; 2006.

19. Kim S. A study on the relationship between personality, study satisfaction and academic achievement of medical students. Korean J Med Educ 1999; 11: 271-284. 
20. Park HG, Lee C, Han OS. Medical specialty preferences and Jung's psychological types of the medical students. J Korean Neuropsychiatr Assoc 1994; 33: 1370-1377.

21. Ahn D, Kim O. Perfectionism, achievement goals, and academic efficacy in medical students. Korean J Med Educ 2006; 18: 141-152.

22. Kim EK, Yang EB, Kim SJ. Exploring the characteristics of interpersonal problems based on medical students' personality types. Korean J Med Educ 2007; 19: 143-151.

23. Jun JY, Kim SA, Moon DS, Kang DW, Park WS. An analysis of influencing factors on the medical students' stress. Kwandong Med J 2000; 4: 127-135.

24. Kim JY, Mang SH, Moon OR, Yoo CA, Lee SJ, Lee SS, Hyun MS. The adaptive behavior of medical students to anatomical dissection. J Ewha Med Assoc 1977; 9: 42-53.

25. Ahn JY. Comparison to health promoting behaviors and perception of sex among women student [master's thesis]. [Seoul, Korea]: Korea University; 2008.

26. Roh MS, Jeon HJ, Kim H, Cho HJ, Han SK, Hahm BJ. Factors influencing treatment for depression among medical students: a nationwide sample in South Korea. Med Educ 2009; 43: 133-139.

27. Min SK, Shin WC, Kim KI, Chung JI, Kim DK. Comparison of quality of life between medical students and general college students. J Korean Neuropsychiatr Assoc 2000; 39: 1054-1060.

28. Ko J, Yoon TY, Park J. The effects of coping style on subjective well-being among medical students. Korean J Med Educ 2007; 19: 225-233.

29. Jung OB. Adolescence psychology. Seoul, Korea: Hakjisa; 2005.

30. Rho MR, Yeom YL. MMPI profile in college student. Soonchunhyang Univ Hum Sci Discuss 1996; 2: 169-175.

31. Kang PS, Kim SB, Hwang TY. Premedical students' motivations for a career as a physician. Korean J Med Educ 1994; 6: 9-19.

32. Kim MK, Kang JO. Comparison of career choice motiva- tion and moral reasoning ability between students in baccalaureate and graduate-entry programs. Korean J Med Educ 2007; 19: 91-99.

33. Kim JY, Son HJ, Kim TJ, Choi YH, Kim HJ, Kee CW, Kim JH, Hong KP. The impact of application motives on medical school adjustment. Korean J Med Educ 2004; 16: $207-218$.

34. Park JY. A study on factors influencing Korean medical students' quality of life [master's thesis]. [Seoul, Korea]: Yonsei University; 2006.

35. Leem TG, Kim DY. Study on students' campus life satisfaction: the case of College of Medicine, Inje University. Inje J 2005; 20: 237-259.

36. Kim YS, Park JW, Son YJ. A longitudinal study on moral judgment development in nursing and medical students. J Korean Acad Nurs 2003; 33: 820-828.

37. Lee JH, Moon YL, Kim JY, Son HJ, Hong KP. A study of comparison between moral sensitivity and reasoning in medical students. Korean J Med Educ 2006; 18: 41-54.

38. Han DS, Cho BH, Bae S, Kim CY, Lee SI, Lee YJ. Professional socialization of medical students. Korean J Prev Med 1996; 29: 265-276.

39. Han JJ, Lee SN, Kwon I, Park H, Im HJ, Kim TE, An $\mathrm{JH}$. The comparison of backgrounds and characteristics of students in medical college and graduate medical school: a case study of one medical school. Korean J Med Educ 2008; 20: 11-21.

40. Hur Y, Lee SH, Kim S. Medical students' self assessment on medical professionalism. Korean J Med Educ 2008; 20: 23-35.

41. Bang JB. A study on the curriculum for the basic quality of medical professionalism [dissertation]. [Gyeongsan, Korea]: Yeungnam University; 2009.

42. Taylor IM, Ntoumanis N, Standage M. A self-determination theory approach to understanding the antecedents of teachers' motivational strategies in physical education. J Sport Exerc Psychol 2008; 30: 75-94. 
Appendix 1. Objects of Analysis

\begin{tabular}{|c|c|c|c|c|}
\hline 분류 & 제목 & 이름 & 연도 & 출처 \\
\hline \multirow{14}{*}{$\begin{array}{l}\text { 정서 } \\
\quad \cdot \\
\text { 적응 } \\
\text { (14편) }\end{array}$} & $\begin{array}{l}\text { 해부실습이 의대생에게 미치는 심리적 영향과 극복과정에 대한 } \\
\text { 연구 }\end{array}$ & 김자예 외 & 1977 & 이화의학회지 1977; 9: 42-53. \\
\hline & 대구지역 의예과 학생들의 의과대학 지망동기에 대한 조사 & 강복수 외 & 1994 & 한국의학교육 1994; 6: 9-19 \\
\hline & 대학생의 MMPI 프로파일 특성 & 노명래 외 & 1996 & $\begin{array}{l}\text { 순천향인문과학논총 } \\
\text { 1996; 2: 169-175. }\end{array}$ \\
\hline & 의과대학생과 일반대학생의 삶의 질 비교 & 민성길 외 & 2000 & $\begin{array}{l}\text { 신경정신의학 } \\
\text { 2000; 39: 1054-1060. }\end{array}$ \\
\hline & 의대생들의 대학생활 만족도에 관한 연구 & 임태균 외 & 2005 & 인제논총 2005; 20: 237-259. \\
\hline & 지원동기가 의과대학 적응에 미치는 영향 & 김지영 외 & 2004 & 한국의학교육 2004; 16: 207-218. \\
\hline & 의대생의 자기효능감과 환경요인, 성격, 학업성취도 관련성 & 이영미 외 & 2006 & 한국의학교육 2006; 18: 249-257. \\
\hline & $\begin{array}{l}\text { 의과대학생들의 완벽주의, 성취목적 및 학문적 효능감 간의 } \\
\text { 관계 }\end{array}$ & 안도희 외 & 2006 & 한국의학교육 2006; 18: 141-152. \\
\hline & $\begin{array}{l}\text { 의과대학과 의학전문대학원 학생들의 진로 선택동기 및 도덕 } \\
\text { 판단력 비교 }\end{array}$ & 김민강 외 & 2007 & 한국의학교육 2007; 19: 91-99. \\
\hline & 의과대학 학생의 성격유형에 따른 대인관계 문제 특성 & 김은경 외 & 2007 & 한국의학교육 2007; 19: 143-151. \\
\hline & $\begin{array}{l}\text { 스트레스 대처방식이 의과대학생들의 주관적 안녕감에 미치는 } \\
\text { 영향 }\end{array}$ & 고진경 외 & 2007 & 한국의학교육 2007; 19: 225-233. \\
\hline & 의대생들의 우울증 치료에 영향을 미치는 요인: 종단적 연구 & 노명선 외 & 2009 & $\begin{array}{l}\text { Medical Education } \\
\text { 2009; 43: 133-139. }\end{array}$ \\
\hline & $\begin{array}{l}\text { 지각된 부모의 학업지원, 성취기대, 일상통제가 학업성취 수준 } \\
\text { 에 따라 자기결정성 동기에 미치는 영향 }\end{array}$ & 신종호 외 & 2010 & 교육심리연구 2010; 24: 121-137. \\
\hline & $\begin{array}{l}\text { 의과대학에서의 교육풍토, 자기주도학습, 그리고 창의적 사고 } \\
\text { 에 대한 고찰-타대학 유사전공 학생들과의 비교를 기반으로 }\end{array}$ & 천경희 외 & 2010 & 사고개발 2010; 6: 179-200. \\
\hline \multirow{13}{*}{$\begin{array}{l}\text { 인지 } \\
\cdot \cdot \\
\text { 학업 } \\
\text { (13편) }\end{array}$} & 의과대학생들의 학업성취 관련요인 코호트 분석 & 유승흠 & 1994 & 한국의학교육 1994; 6: 20-28. \\
\hline & 시험 스트레스가 의대생의 면역기능에 미치는 영향 & 김충수 외 & 1995 & 충남의대잡지 1995; 22: 423-436. \\
\hline & $\begin{array}{l}\text { 의과대학생의 성격과 학업만족도 및 학업성취도간의 관련성 } \\
\text { 연구 }\end{array}$ & 김선 & 1999 & 한국의학교육 1999; 11: 271-284. \\
\hline & 의과대학생의 학습양식의 특성과 학업성취와의 관계 & 김미라 외 & 2006 & 한국의학교육 2006; 18: 133-140. \\
\hline & $\begin{array}{l}\text { 의과생과 한의대생의 보완대체의료에 대한 지식, 태도, 신념에 } \\
\text { 관한 연구 }\end{array}$ & 김향자 & 2006 & 서울대학교 보건대학원 학위논문 \\
\hline & 한국 의대생의 삶의 질에 영향을 미치는 요인 연구 & 박진영 & 2006 & 연세대학교 대학원 학위논문 \\
\hline & 일개 의과대학 의학과 학생의 학습양식 & 여상희 외 & 2006 & 한국의학교육 2006; 18: 31-40. \\
\hline & Kolb 학습양식 검사지를 이용한 의과대학생의 학습양식분석 & 김상현 외 & 2007 & 한국의학교육 2007; 19: 197-205. \\
\hline & $\begin{array}{l}\text { 의과대학생들의 학업동기, 학업스트레스 및 학업성적에 대한 } \\
\text { 지각 간의 관계 }\end{array}$ & 안도희 외 & 2007 & 한국의학교육 2007; 19: 59-71. \\
\hline & $\begin{array}{l}\text { 의과대학생들의 성취목표성지향성과 자기효능감이 수업에 대 } \\
\text { 한 흥미와 학업성취도에 미치는 영향 }\end{array}$ & 소연희 & 2008 & 한국의학교육 2008; 20: 37-49. \\
\hline & $\begin{array}{l}\text { 의과 대학생들의 지적능력에 대한 신념을 예측하는 변인 탐색: } \\
\text { 인지적·정의적 특성, 부모의 자녀교육관심도 및 가정의 경제적 } \\
\text { 수준 }\end{array}$ & 안도희 외 & 2008 & 한국의학교육 2008; 20: 219-229. \\
\hline & $\begin{array}{l}\text { 충북대학교 의학전문대학원 학생들의 특성 및 인지적 입학전형 } \\
\text { 요소와 학업성취도의 관계 }\end{array}$ & 이상진 외 & 2009 & $\begin{array}{l}\text { Korean Journal of Medical Education } \\
\text { 2009; 21: } 59-66 \text {. }\end{array}$ \\
\hline & 의예과 학생들의 인지양식과 학습양식의 관계 & 안서원 외 & 2010 & $\begin{array}{l}\text { Korean Journal of Medical Education } \\
\text { 2010; 22: 7-13. }\end{array}$ \\
\hline
\end{tabular}


류숙희, 이혜범 : 국내 의학과, 의학전문대학원, 의예과 학생들의 인지·정서 - 사회적 특성 고찰

\begin{tabular}{clccc}
\hline 분류 & \multicolumn{1}{c}{ 제목 } & 이름 & 연도 & 출처 \\
\hline \multirow{4}{*}{ 의대생의 전문직 사회화 과정에 대한 고찰 } & 한달선 외 & 1996 & 예방의학회지 1996; 29: 265-276. \\
& 의대생의 스트레스에 영향을 미치는 요인 분석 & 전진용 외 & 2000 & 관동의대학술지 2000; 4: 127-135. \\
& 간호대생과 의대생의 도덕 판단력 발달에 관한 종단적 연구 & 김용순 외 & 2003 & 대한간호학회지 2003; 33: 820-828. \\
& 의과대학생의 도덕민감성과 도덕 판단력의 비교 연구 & 이지혜 외 & 2006 & 한국의학교육 2006; 18: 41-54. \\
사회 & 여대생의 건강증진행태와 성인식에 관한 비교연구 & 안지연 & 2008 & 고려대학교 보건대학원 학위논문 \\
도덕성 & 의과대학생의 의학 전문 직업성 수준에 대한 학생과 교수의 & 허예라 & 2008 & 한국의학교육 2008; 20: 209-217. \\
(9편) & 인식 차이 & & & \\
& 의과대학생들의 의학전문 직업성에 대한 자기평가 & 허예라 외 & 2008 & 한국의학교육 2008; 20: 23-35. \\
& 의과대학과 의학전문대학원 신입생의 배경 및 특성 비교: 일개 & 한재진 외 & 2008 & 한국의학교육 2008; 20: 11-21. \\
& 대학 사례 & & & \\
& 의학 전문 직업성의 기초자질 함양을 위한 교육과정 탐색 & 방재범 & 2009 & 영남대학교 대학원 학위논문 \\
\hline
\end{tabular}

